\title{
Quantifying the Spatiotemporal Influence of Acute Myocardial Ischemia on Volumetric Conduction Speeds
}

\author{
Wilson W Good ${ }^{1,2,3}$, Brian Zenger ${ }^{1,2,3,4}$, Jake A Bergquist ${ }^{1,2,3}$, \\ Lindsay C Rupp ${ }^{1,2,3}$, Karli Gillette ${ }^{5}$, Gernot Plank ${ }^{5}$, Rob S MacLeod ${ }^{1,2,3}$ \\ ${ }^{1}$ Scientific Computing and Imaging Institute, University of Utah, SLC, UT, USA \\ ${ }^{2}$ Department of Biomedical Engineering, University of Utah, SLC, UT, USA \\ ${ }^{3}$ Nora Eccles Cardiovascular Research and Training Institute, University of Utah, SLC, UT, USA \\ ${ }^{4}$ School of Medicine, University of Utah, SLC, UT, USA \\ ${ }^{5}$ Institute of Biophysics, Medical University of Graz, Graz, Austria
}

\begin{abstract}
Acute myocardial ischemia compromises the ordered electrical activation of the heart, however, because of sampling limitations, volumetric changes in activation have not been measured. We used a large-animal experimental model and high-resolution volumetric mapping to study the effects of ischemia on conduction speeds (CS) throughout the myocardium. We estimated CS and electrocardiographic changes (ST segments) and evaluated the spatial and temporal correlations between them across 11 controlled episodes. We found that ischemia induces significant conduction slowing, reducing the global median speed by $25 \mathrm{~cm} / \mathrm{s}$. Furthermore, there was a high temporal correlation between the development of ischemic severity and CS (corr. = 0.93) through each episode. The spatial correlations between ST-segment changes and CS slowing were more spatially complex than expected with substantial slowing at the periphery of the zones that showed ST-segment changes. This is the first study that has documented in an experimental model volumetric changes of CS during acute myocardial ischemia and explored the relationships between ischemia development in space and time. We showed that conduction speed changes are spatiotemporally correlated to ischemic severity and illustrated the biphasic response long proposed from cellular studies.
\end{abstract}

\section{Introduction}

Myocardial ischemia occurs when coronary perfusion to the heart is inadequate. This perfusion shortfall initiates an electrochemical cascade known to influence the ST and repolarization phases of the ECG but it also has a marked effect on propagation (QRS).[1] These effects are thought to drive several time periods during acute myocardial ischemia that are arrhythmogenic, which can lead to sudden cardiac death (SCD).[2] These effects are highly dynamic and spatially complex and they occur within the ventricular walls, making their experimental study especially challenging. Such studies require intramural sampling to measure volumetric conduction speed and study propagation during episodes of controlled ischemic stress.

Previous studies have shown the feasibility of volumetric measurements using intramural plunge needles to quantify the spatial extent and severity of ischemic stress based on electrocardiographic parameters, typically ST-segment shifts. $[3,4]$ CS changes in the presence of acute ischemia have also been documented most extensively during cellular studies. [1] These cellular studies also showed a biphasic response in excitability (increase followed by decrease) of the tissue early in the ischemic evolution, but the anticipated changes in CS also have not been replicated.

Conduction speed must be estimated from spatially distributed measurements and published studies have so far been limited to surface measurements.[5] We have created and validated a novel volumetric CS estimation technique based on intramural measurements and demonstrated its accuracy.[6] Applying this method to measurements made during our experiments was the logical next step in the study of arrhythmogenesis during acute ischemia.

This study examined the spatiotemporal relationship between the intramural development of ischemia as detected through ST-segment shifts and changes in conduction speed. We identified high temporal correlations between ischemic stress and CS slowing. Less anticipated were the complex spatial relationships we documented between CS slowing and regions of ischemic stress.

\section{Methods}

Experimental Preparation: Signals were acquired from two in situ canine experiments as described previ- 
ously. [3, 4] In brief, each experiment consisted of 5-6 episodes of controlled acute myocardial ischemia. Hearts were then removed and imaged using high-resolution MRI and geometric models were created that merged anatomy with electrode positions, as described previously. $[4,7,8]$

Estimating Volumetric Conduction Velocity: To estimate volumetric conduction velocity, we (1) reconstructed the activation wavefront,[6] (2) calculated the elementwise gradient to approximate propagation direction, and (3) estimated conduction speed with an inverse-gradient technique.[9] All steps were based on activation times estimated from electrograms recorded with intramural plunge needle arrays. Higher resolution activation times were then reconstructed on the nodes of the geometric model of the heart using radial basis interpolation. The geometric model was then clipped by the outer bounds of the measurement electrodes and the blood pool, leaving only the region sampled by the intramural plunge needles, which we refer to as the 'needle envelope'. We estimated the wavefront direction by calculating the direction of the gradient of activation times throughout the needle envelope. We estimated conduction velocity using an inverse-gradient technique, which inverts the activation gradient and takes its partial derivative on an element-wise basis. [6,9] The result is a conduction velocity vector assigned to each element in the geometric model from which we computed CS.

Quantifying CS Changes During Ischemia: CS and ST40\% distributions were calculated throughout the 11 ischemic episodes. For each episode, a continuous 3second 'run' was captured every 15 seconds and a representative beat selected from each run, resulting in 25-35 beats per episode. The ST40\% was defined as the potential value $40 \%$ between the end of the QRS and the peak of the T-wave.[3] Measured ST40\% values were interpolated into the needle envelope using the same radial basis interpolation scheme described above. We compared the CS and ST40\% values before the induction of ischemia with those at the peak of each ischemic episode. We evaluated the differences in the skewed distributions using a two-sample Kolmogorov-Smirnov test (KS-test) and the episodic changes were determined to be significant when the p-value was less than 0.05 .

Temporal Examination of Conduction Changes: To determine the temporal relationship between CS slowing and ST40\% development, we measured the temporal correlation of median ST40\% and median CS values throughout the ischemic episodes. Medians were computed across all nodes and for each representative beat within an episode of ischemia to form a 'run-metric plot' (e.g., Figure 2B), showing the CS and ST40\% evolution over time.

Spatial Examination of Conduction Changes: We performed a spatial analysis to determine if different levels of ischemic stress as quantified by ST40\% values influence changes in CS. We analyzed the ST40\% distribution at the peak of each episode and found the regions within the needle envelope with ST40\% values in the lowest and the highest quartiles. We then calculated the relative change of CS in each of the identified regions during peak ischemia. Statistically significant differences were determined using a Student $t$-test with $\mathrm{p}<0.05$. The Student $\mathrm{t}$-test was used to detect whether the changes in the two cohorts across the 11 episodes of ischemia were significant and the distribution of changes were normal.

\section{Results}

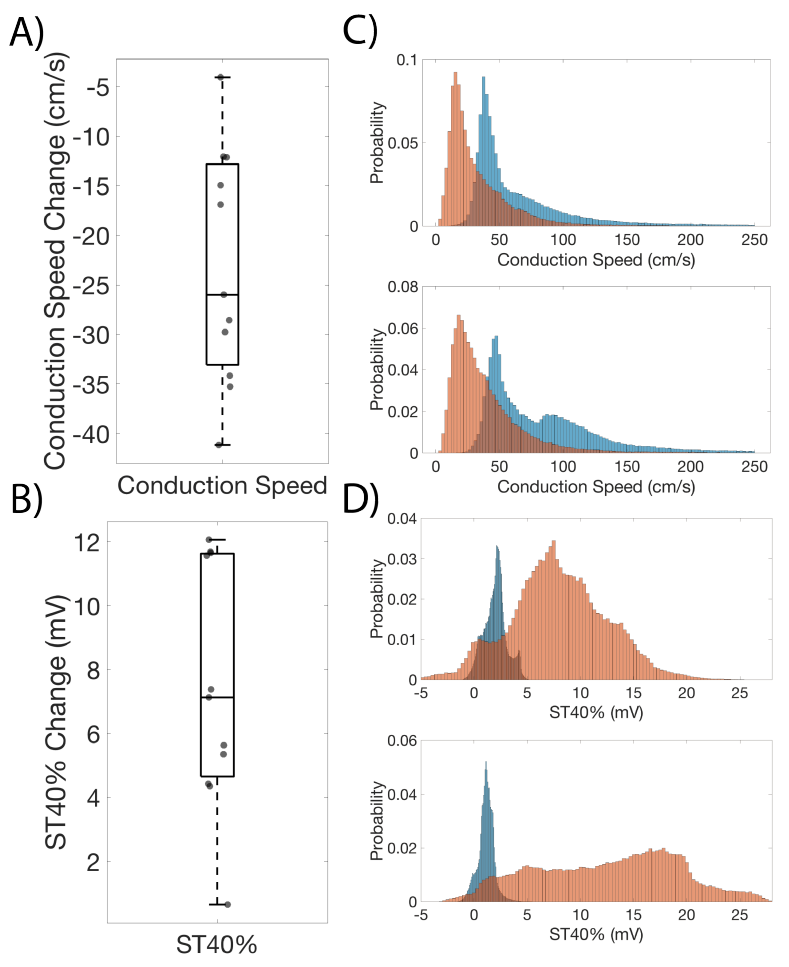

Figure 1. Changes in CS and ST40\% distributions between peak and control periods of the ischemic episodes. A) The change in median CS across 11 episodes of ischemia. B) The change in median ST40\% across 11 episodes of ischemia. C) Two sample CS distributions prior to the induction of ischemia (blue) and at the peak (orange) of the ischemic episodes. D) ST40\% distributions for the same episodes as in Panel C.

Quantifying the Global Conduction Speed Changes: Figure 1A and Figure 1B show the median ST40\% and CS changes between peak and control periods across the 11 episodes of ischemia. Figure 1C shows two sample distributions of CS at control (blue) and the peak of the ischemic episodes (orange). Similarly, Figure 1D shows the ST40\% distributions for the same episodes. The median drop in CS was approximate $25 \mathrm{~cm} / \mathrm{s}$ across the 11 episodes. Median ST40\% changes were approximately $7 \mathrm{mV}$. The CS distributions at control and peak across the 11 episodes were 
each determined to be significantly different $(\mathrm{p}<0.05)$.

Temporal Examination of Conduction Changes: Figure $2 \mathrm{~A}$ shows the measured temporal correlation between CS and ST40\% across the 11 episodes with a median correlation of 0.93 . Figure $2 \mathrm{~B}$ shows examples of temporal changes of the median CS slowing and ST40\% over three ischemic episodes from early, middle and late in a single experiment. These episodes were representative of a general trend: CS slowing typically lagged behind the elevation of ST40\% early in the experiment, whereas in the later episodes, CS changes typically preceded ST40\% changes. Another interesting result observed across both experiments was a biphasic response in CS (e.g., Figure 2B) during episodes induced near the middle of the experiment.)
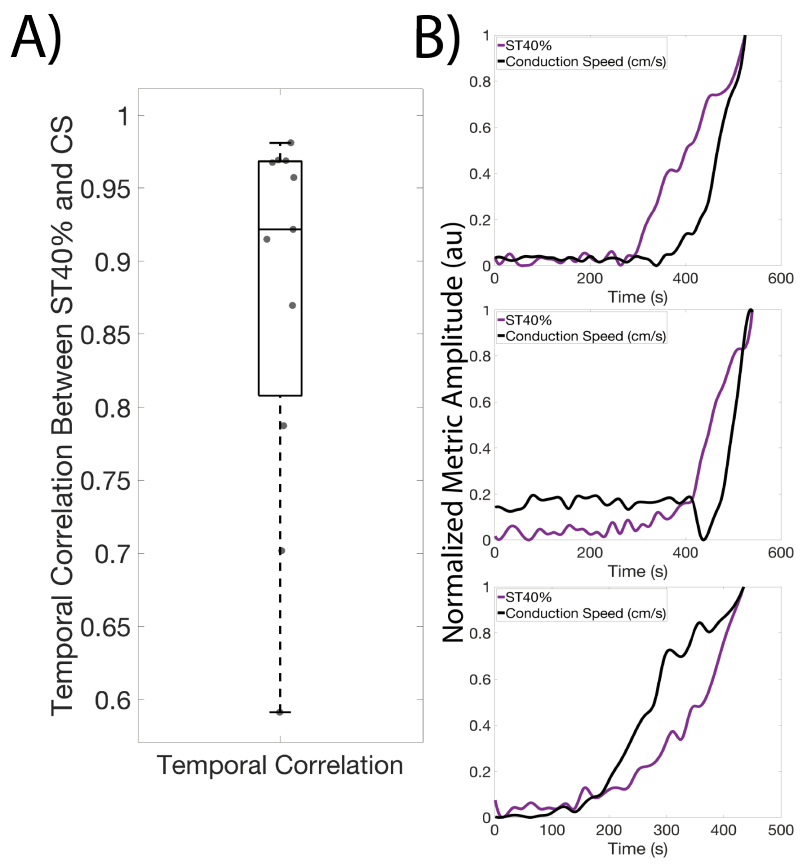

Figure 2. Temporal evolution of ST40\% and CS slowing during ischemia. A) The temporal correlation between the decrease in CS and increase in ST40\% across 11 episodes of ischemia. B) Three examples of the normalized values of CS slowing and ST40\% during the ischemic episode shown as a run-metric plot. Episodes from early (Top), middle (Middle) and near the end of the experiment (Bottom).

Spatial Examination of Conduction Changes: Figure 3 contain results from an analysis of spatial overlap between ST40\% and CS. Panel A shows that significantly larger conduction slowing occurs only in the most severe ischemic tissue. Panel B shows the intramural needles (grey spheres) within the myocardium. Panel C shows the spatial distribution of the regions of extreme ST-elevation (blue) overlaying regions of extreme conduction slowing (red), showing a complex spatial relationship between the two volumes.

\section{Discussion}

This study has shown for the first time the utility of using volumetric estimations of CS to study propagation changes during acute myocardial ischemia. We were able to reconstruct the spread of activation with adequate resolution to compare the temporal and spatial correlations between CS slowing and ST40\% elevation through 11 different episodes of controlled ischemia in 2 experiments using a canine model.

We found significant conduction slowing that occurred during acute ischemia within a needle envelope located in the putative perfusion zone. While our intramural measurements could only include part of the left ventricle, we replicated previous studies and identified localized regions of ST-segment elevation that arise during acute ischemia [3] In addition, we were able for the first time to examine changes in both ST-segment potentials and conduction speed. We showed that the time course of conduction slowing was correlated with an increase in ST40\%, which we associated with the severity of ischemia. A further notable temporal response was the paradoxical appearance of transient increases in CS early in an ischemia episode. During four of the ischemic episodes across both experiments, we observed such biphasic responses in CS. Qualitative examination suggests this early quickening happens in the less severely ischemic tissue, supporting observations made decades ago in cellular studies.[1]

A parallel comparison of the spatial progress of ST40\% and CS was much more complex than the temporal correlation but also consistent enough to suggest some systematic observations. For example, we observed the largest absolute changes in CS within the subendocardium, where the CS was fastest at control. Ischemia clearly has a widespread impact on CS but only at the extremes does it show correlation with changes in ST40\%. Contrary to our expectations, regions of high ST40\% elevation did not overlap consistently with CS slowing. Instead, regions of slowed CS were often at the edges of the zones with high levels of ischemia.

The combination of spatial heterogeneity of CS and its temporal progression naturally suggest speculations related to the arrhythmogenesis that previous studies have shown occurs in early ischemia. Of course, such speculation must also include information about repolarization, the parameters of which are also available from our studies. It is essential to uncover regions of functional unidirectional block that our ongoing studies will seek to identify.

Our preparation shares the limitation of all mapping studies in that spatial sampling and coverage are a compromise between achievable electrode densities and maintaining a viable, living organ under physiological conditions. Our sampling is relatively dense in the radial direction $(1.5-1.7 \mathrm{~mm})$, which is ideal for studying endocardial 

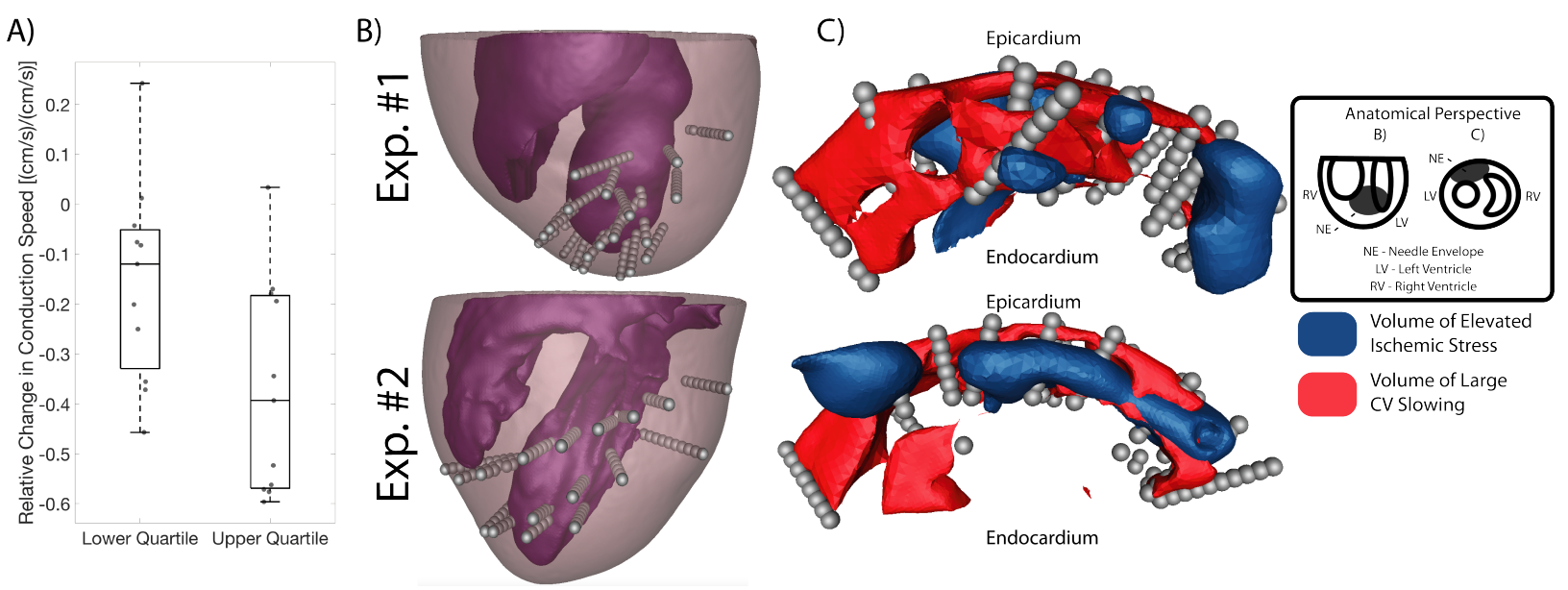

Figure 3. Spatial distribution of high ischemic stress (blue) and large changes in CS (red). A) The distribution of relative CS changes for the nodes in the lower quartile and upper quartiles of the ST40\% changes. B) Anatomy of the two hearts used in this study showing the intramural needle (grey spheres) distribution relative to the ventricular chambers (in purple). C) Overlay of regions of large ST40\% elevation (blue) and conduction slowing (red) with the needles envelopes from the heart in Panel B.

to epicardial gradients. Sampling in orthogonal directions is more limited $(1-3 \mathrm{~cm})$, making spatial examination in these directions more prone to error. However, our validation studies suggest that even in these relatively sparsely sampled regions, the $\mathrm{CV}$ can be estimated with high fidelity.[6]

This study is the first to report, from experiments, volumetric conduction speed changes during episodes of acute ischemia. We showed that conduction speed changes were temporally correlated to ischemic severity and illustrated the biphasic response long proposed from cellular studies. Furthermore, our results suggest extreme conduction slowing in regions of severe ischemia but that the overall relationship between ischemia and CS is more complex than previously anticipated.

\section{Acknowledgments}

Support for this research comes from the NIH NIGMS Center for Integrative Biomedical Computing (www.sci.utah.edu/cibc), NIH NIGMS grants no. P41GM103545 and R24 GM136986., the Nora Eccles Treadwell Foundation for Cardiovascular Research.

\section{References}

[1] Elharrar V, Zipes D. Cardiac electrophysiologic alterations during myocardial ischemia. American Journal of Physiology Heart and Circulatory Physiology 1977;233(3):H329H345.

[2] Stern S. State of the art in stress testing and ischaemia monitoring. Cardiac Electrophysiology Review 2002;6(3):204208.

[3] Aras K, Burton B, Swenson D, MacLeod R. Spatial organization of acute myocardial ischemia. Journal of Electrocardiology 2016;49(3):323-336.
[4] Zenger B, Good WW, Bergquist JA, Burton BM, Tate JD, Berkenbile L, Sharma V, MacLeod RS. Novel experimental model for studying the spatiotemporal electrical signature of acute myocardial ischemia: a translational platform. Physiological Measurement 2020;41(1):015002.

[5] Cantwell CD, Roney CH, Ng FS, Siggers JH, Sherwin S, Peters NS. Techniques for automated local activation time annotation and conduction velocity estimation in cardiac mapping. Computers in Biology and Medicine 2015;65:229-242.

[6] Good WW, Gillette K, Bergquist JA, Zenger B, Rupp LC, Tate J, Anderson D, Plank G, , MacLeod RS. Estimation and validation of cardiac conduction velocity and wavefront reconstruction using epicardial and volumetric data. IEEE Transactions in Biomedical Engineering 2020; In Preparation.

[7] Bergquist JA, Good WW, Zenger B, Tate JD, MacLeod. RS. GRÖMeR: A pipeline for geodesic refinement of mesh registration. In Lecture Notes in Computer Science, volume 11504. Functional Imaging and Model of the Heart (FIMH), Springer Verlag, 2019; 37-45.

[8] Neic A, Gsell MA, Karabelas E, Prassl AJ, Plank G. Automating image-based mesh generation and manipulation tasks in cardiac modeling workflows using meshtool. SoftwareX 2020;11:100454.

[9] Bayly PV, KenKnight BH, Rogers JM, Hillsley RE, Ideker RE, Smith WM. Estimation of conduction velocity vector fields from epicardial mapping data. IEEE Transactions in Biomedical Engineering 1998;45(5):563-571.

Address for correspondence:

Name: Wilson William Good

Full postal address: SCI Institute, University of Utah, 72 Central Campus Dr. Room 3750, Salt Lake City, UT 84112

E-mail address: wgood@sci.utah.edu 